Japanese Journal of Physiology, 30, 655-658, 1980

\title{
Incremental Conduction Velocity of Single Afferent Fibers Innervating Frog Taste Organ
}

\author{
Toshihide SAto,* Mitsutaka Ohkusa, and Kumiko Sugimoto \\ Department of Physiology, Faculty of Dentistry, Tokyo Medical and \\ Dental University, Tokyo, 113 Japan
}

\begin{abstract}
Summary The mean diameter of myelinated afferent fibers from the taste disk located on the frog tongue was $5.5 \mu \mathrm{m}$ within the fungiform papillae and increased at a rate of $0.8 \mu \mathrm{m} / \mathrm{cm}$ length of fibers. A gradual increase in conduction velocity of single afferent fibers from the taste disk was confirmed electrophysiologically.
\end{abstract}

There is a possibility that in the peripheral sensory nervous system, the diameter of single afferent fibers increases gradually as they run centrally. Hence, it is likely that the conduction velocities for single sensory fibers increase progressively.

The purpose of the present work is to clarify this possibility morphologically and physiologically using myelinated afferent fibers which originate from the taste disk of the frog tongue.

All the experiments were carried out with adult bullfrogs (Rana catesbeiana) at a room temperature of about $25^{\circ} \mathrm{C}$. When measuring fiber diameters, an isolated tongue including the glossopharyngeal nerves was stained with $0.01-0.1 \%$ methylene blue in a frog Ringer solution. The outer diameters (axon plus myelin sheath) of the stained fibers were directly observed with a light microscope without any other histological procedures.

To measure the conduction velocity of an impulse of a single myelinated afferent fiber from the taste disk located on the tongue, a main lingual branch of the glossopharyngeal nerve, attached to a small segment of the apical tongue area, was dissected from the animal after the other parts of the tongue and many small lingual branches of the nerve within the tongue had been removed (see the upper inset in Fig. 2). The two points $6 \mathrm{~mm}$ apart in the glossopharyngeal nerve bundle were individually stimulated by single cathodal current pulses of

Received for publication February 9, 1980

* present address: Department of Physiology, Nagasaki University School of Dentistry, 7-1 Sakamoto-machi, Nagasaki, 852 Japan

Requests for reprints to Dr. T. Sato at the present address.

佐藤俊英, 大草光高, 杉本久美子 

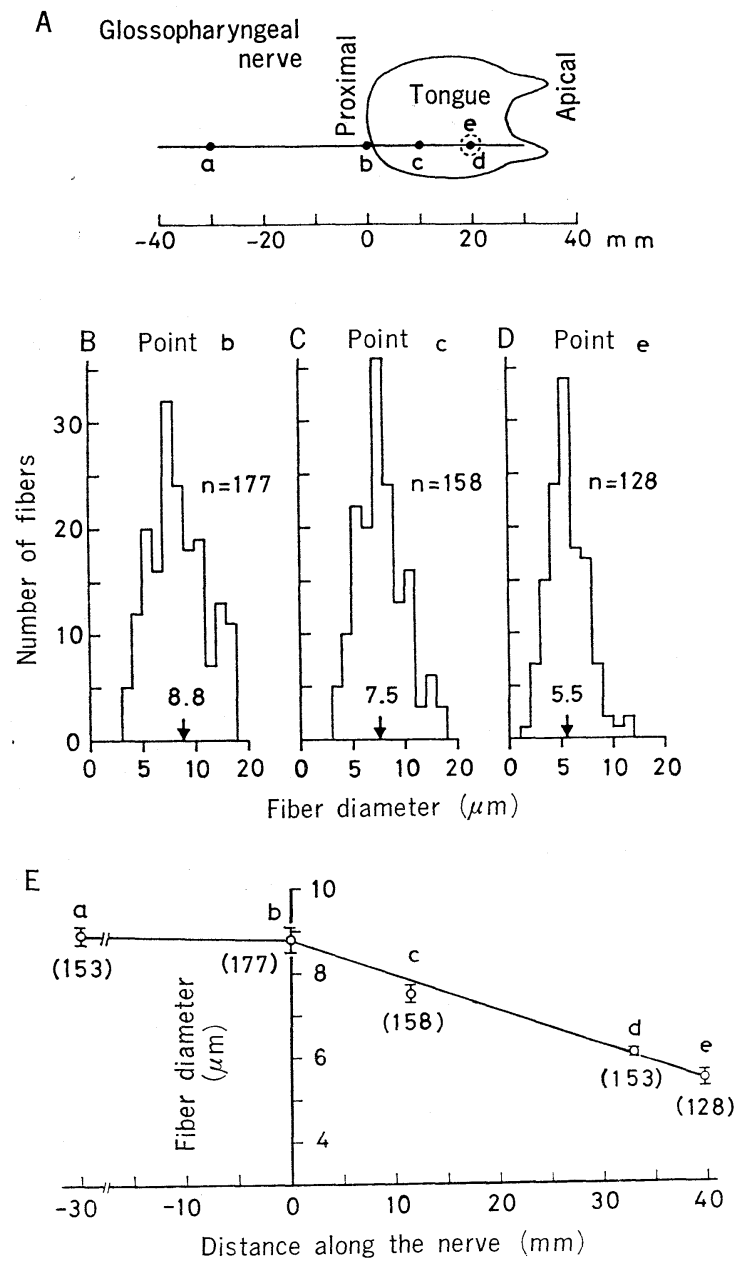

Fig. 1. A: schematic diagram of the tongue with an attached glossopharyngeal nerve. The tongue at rest, not stretched, and the main nerve trunk alone are illustrated. The four solid circles $(a, b, c$ and $d$ ) denote the points along the nerve where fiber diameters were measured. The one larger interrupted circle $(e)$ denotes the dorsal tongue surface area of $9 \mathrm{~mm}^{2}$ lying across the point $d$, where the diameter of fibers entering the fungiform paipllae was measured. The proximal end of the tongue was taken as $0 \mathrm{~mm}$. B, C and $\mathrm{D}$ : fiber diameter histograms obtained from points $b(\mathrm{~B}), c(\mathrm{C})$ and $e(\mathrm{D})$. The ordinate indicates the number of randomly sampled fibers. The arrows show mean value. E: relation between straight distances along the nerve from the reference point $(0 \mathrm{~mm})$ and fiber diameters. The vertical bars show SE. The symbols $a-e$ correspond to those in Fig. 1A, and numerals within parentheses show the number of fibers sampled at each point. 
threshold intensity and $0.1 \mathrm{msec}$ duration while recording antidromic action potentials of a lowest threshold sensory fiber from a fungiform papilla, which was drawn into a recording suction electrode (SATO, 1978). The velocities of an impulse conducted along every $6 \mathrm{~mm}$ distance of a single afferent fiber originating from the taste disk were calculated by dividing the conduction distance of $6 \mathrm{~mm}$ by the difference between conduction times of impulse.

Figure 1A shows a diagram of the frog tongue at rest together with a glossopharyngeal nerve. The four solid circles $(a-d)$ along the nerve illustrate the measuring points of fiber diameters, and the one larger interrupted circle $(e)$ shows the dorsal tongue surface of $9 \mathrm{~mm}^{2}$ lying just across the point $d$ situated ventrally, where the fiber diameter within the fungiform papillae was measured. The proximal edge of the tongue was taken as a reference point, $0 \mathrm{~mm}$. Figures 1B,

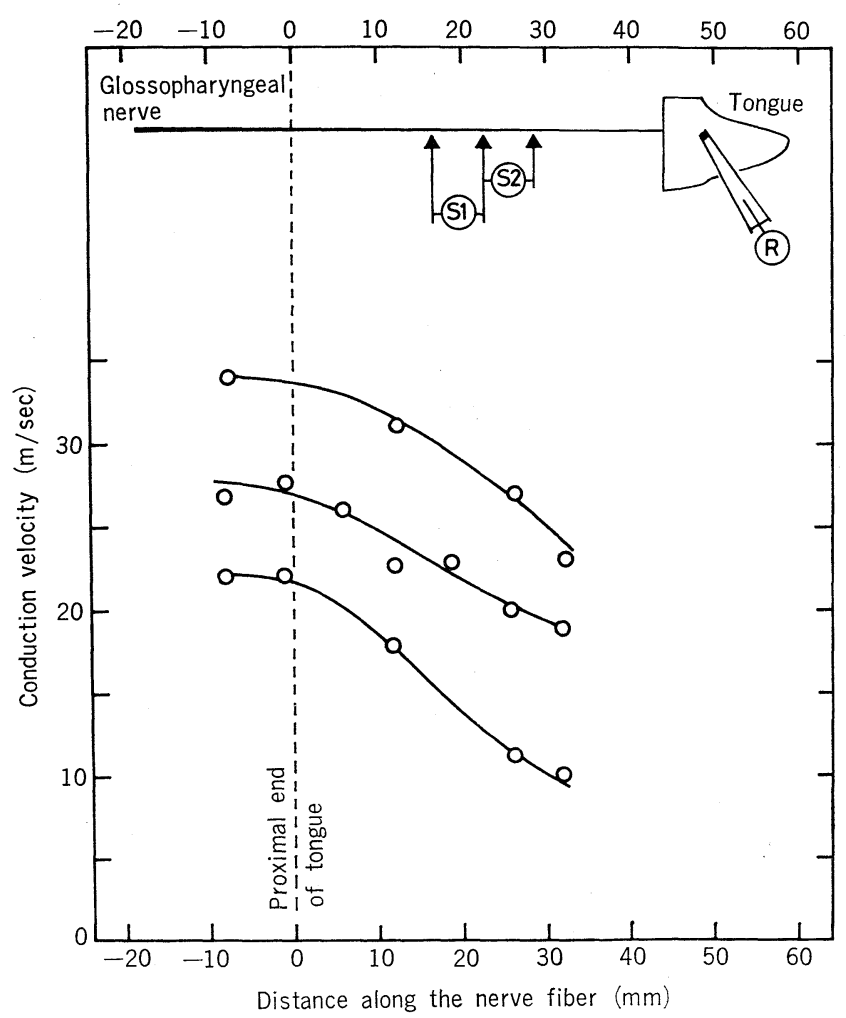

Fig. 2. Conduction velocity of single myelinated afferent fibers from the taste disk on the frog tongue as a function of distance along the fiber from the proximal end of the tongue. The vertical dashed line indicates the proximal end of the tongue taken as $0 \mathrm{~mm}$. In the upper inset are shown a schematic diagram of the preparation and the arrangement of stimulating and recording electrodes. S1 and S2: electrical stimulation of the nerve with $0.1 \mathrm{msec}$ pulses through silver wires. $\mathrm{R}$ : recording from a fungiform papilla with a Ringer-filled suction electrode. 
C, and D illustrate histograms of the diameters of myelinated afferent fibers measured at the points $b, c$ and $e$ in Fig. 1A, respectively. Due to technical limitations we could not find nonmyelinated fibers, the existence of which has been reported elsewhere (KUTYNA and BERNARD, 1977).

The afferent nerve runs spirally throughout the tongue at rest so that the distance between two points on the resting tongue does not indicate the real length of the nerve running within the tongue. Morphologically determined straight distances in the nerve between the proximal end of the tongue and each measured point in Fig. 1A are given in the abscissa of Fig. 1E. It is seen that the mean fiber diameter increases proportionally as the nerve travels from the point $e$ towards the proximal portion of the tongue, but it does not alter after the nerve exits from the tongue. The mean fiber diameter within the tongue increased at a rate of $0.8 \mu \mathrm{m} / \mathrm{cm}$ length of fiber as it runs centrally. This finding may indicate that the conduction velocity for single afferent fibers from the taste disk increases gradually.

To clarify electrophysiologically the problem of incremental conduction velocity, the conduction velocities of single taste disk fibers were measured at various points along the glossopharyngeal nerve. Figure 2 illustrates the change in conduction velocities for three myelinated afferent fibers from the taste disks. The vertical dashed line denoting the proximal end of the tongue separates afferent fibers leaving the tongue (left-hand) from the ones entering the tongue (righthand). It is obvious that conduction velocity of single taste disk fibers within the tongue increases gradually when the impulse is conducted orthodromically.

Earlier measurement of conduction velocities for sensory nerve fibers supplying the frog taste disk indicates that they range from 1 to $15 \mathrm{~m} / \mathrm{sec}$ with a mean of about $5 \mathrm{~m} / \mathrm{sec}$ (RAPUZzI and CASELlA, 1965). Even this maximum value is much smaller than our values $(18,24,32 \mathrm{~m} / \mathrm{sec})$ calculated as the mean within the conduction distance of $40 \mathrm{~mm}$, as shown in Fig. 2 .

A close correlation between the morphological (Fig. 1) and physiological (Fig. 2) data in the present work indicates that the velocity of impulse conducting along a single myelinated afferent fiber changes gradually as a function of fiber diameter as is already well accepted.

We wish to express our thanks to Professor M. Ichioka for his encouragement and Miss M. Oguro for her great help in preparing this article.

\section{REFERENCES}

KutYNA, F. and BernARD, R. A. (1977) Effects of antidromic activity in gustatory nerve fibers on taste disc cells of the frog tongue. J. Comp. Physiol., 118A: 291-306.

Rapuzzi, G. and CASElla, C. (1965) Innervation of the fungiform papillae in the frog tongue. J. Neurophysiol., 28: 154-165.

SATo, T. (1978) Off-response in frog taste nerve and cell after stimulation of the tongue with bitter solutions. Comp. Biochem. Physiol., 61A: 339-353. 